\title{
Fine Structure and Olfactory Reception of the Labial Palps of Spodoptera frugiperda
}

\author{
Qiuyan Chen 1,2, Xiaolan Liu', Song Cao, ${ }^{1,3}$ Baiwei Ma', Mengbo Guo ${ }^{1,3}$, Jie Shen ${ }^{2}$ \\ and Guirong Wang ${ }^{1,3 *}$
}

'State Key Laboratory for Biology of Plant Diseases and Insect Pests, Institute of Plant Protection, Chinese Academy of Agricultural Sciences, Beijing, China, ${ }^{2}$ Department of Entomology and MOA Key Laboratory for Monitory and Green Control of Crop Pest, China Agricultural University, Beijing, China, ${ }^{3}$ Shenzhen Branch, Guangdong Laboratory for Lingnan Modern Agriculture, Genome Analysis Laboratory of the Ministry of Agriculture and Rural Affairs, Agricultural Genomics Institute at Shenzhen, Chinese Academy of Agricultural Sciences, Shenzhen, China

OPEN ACCESS

Edited by:

Peng He,

Guizhou University,

China

Reviewed by:

Hongbo Jiang,

Southwest University,

China

Zhao-Qun Li,

Chinese Academy of Agricultural

Sciences (CAAS), China

Feng Liu,

Michigan State University,

United States

*Correspondence:

Guirong Wang

grwang@ippcaas.cn

Specialty section:

This article was submitted to Invertebrate Physiology, a section of the journal

Frontiers in Physiology

Received: 15 March 2021

Accepted: 24 May 2021

Published: 03 August 2021

Citation:

Chen Q, Liu X, Cao S, Ma B, Guo M,

Shen J and Wang G (2021)

Fine Structure and Olfactory

Reception of the Labial Palps of

Spodoptera frugiperda.

Front. Physiol. 12:680697.

doi: 10.3389/fphys.2021.680697
The olfactory system of insects is essential in many crucial behaviors, such as host seeking, mate recognition, and locating oviposition sites. Lepidopteran moths possess two main olfactory organs, including antennae and labial palps. Compared to antennae, the labial palps are relatively specific and worthy of further investigation due to the labial-palp pit organ (LPO), which contains a large number of sensilla located on the tip segment. The fall armyworm, Spodoptera frugiperda, is a worldwide lepidopteran pest, which can damage more than 350 plants and cause significant economic losses. In this study, we surveyed the structure of the labial palps and LPO of $S$. frugiperda using a super-high magnification lens zoom 3D microscope. Then, the distribution and fine structure of sensilla located in the LPO of $S$. frugiperda were investigated using scanning electron microscopy. Subsequently, the electrophysiological responses of labial palps to $\mathrm{CO}_{2}$ and 29 plant volatiles were recorded by using electrolabialpalpography. Our results showed the fine structure of labial palps, the LPO, and the sensilla located in the LPO of S. frugiperda. Moreover, we demonstrated that the labial palps are olfactory organs that respond to both $\mathrm{CO}_{2}$ and other volatile compounds. Our work established a foundation for further study of the roles of labial palps in insect olfactory related behaviors. Further investigations on the function of labial palps and their biological roles together with $\mathrm{CO}_{2}$ and volatile compound responses in $S$. frugiperda are necessary, as they may provide better insect behavioral regulators for controlling this pest.

Keywords: Spodoptera frugiperda, olfactory, labial-palp pit organ, $\mathrm{CO}_{2}$, volatiles

\section{INTRODUCTION}

The sophisticated olfactory sensing organs of most insects have important roles in detecting host volatiles, recognizing mates, and locating oviposition sites. These organs are mainly distributed in the head, including antennae and mouthpart appendages. As the primary olfactory sensory organs, insect antennae bear abundant of sensilla that are sensitive to plant volatiles, 
sex pheromones, and other volatile components. Additionally, some olfactory sensilla are also found on mouthpart appendages, such as maxillary palps (Syed and Leal, 2007; Bohbot et al., 2014) and labial palps (Stange and Stowe, 1999; Galizia and Rossler, 2010). As an important sensory organ, the well-developed labial palps are located on each side of the proboscis in adult Lepidoptera. The labial-palp pit organ (LPO) is a unique structure of lepidopteran species that is located on the apex of labial palps, within which the sensilla lie.

The labial palps are densely covered with scales and usually contain three segments. If the scales are removed, a bottleshaped LPO situated on the tip of the third segment of the labial palp can be observed. Detailed electron microscopical analyses have been performed on the structure of the LPO in many lepidopteran species (Stange and Stowe, 1999; Faucheux, 2008; Zhao et al., 2013; Dong et al., 2014; Barcaba and Krenn, 2015; Chen and Hua, 2016; Yan et al., 2019), which not only showed large numbers of olfactory sensilla in the LPO but also provided descriptions of the fine structure of LPO and LPO sensilla. The morphological characteristics of LPO and LPO sensilla in adult Lepidoptera are somewhat variable. Usually, the LPO of moths is about 100-300 $\mu \mathrm{m}$ deep and 30-80 $\mu \mathrm{m}$ wide. LPO sensilla can be divided into one to three morphological types. The number of LPO sensilla varies from 80 (Lee et al., 1985) to 1,750 (Kent et al., 1986) in different lepidopteran species.

Compared to antennae, the function of labial palps is largely unknown. At present, the most important function of labial palps in adult Lepidoptera that has been reported is detecting carbon dioxide $\left(\mathrm{CO}_{2}\right)$. Electrophysiological recording preformed on the sensilla in the LPO of butterfly (Lee et al., 1985) and moth (Bogner et al., 1986; Stange et al., 1995; Guerenstein et al., 2004; Ning et al., 2016) all showed that the LPO sensilla react to $\mathrm{CO}_{2} \cdot \mathrm{CO}_{2}$ is a ubiquitous source of ecologically relevant information in insect-plant interactions, insect-vertebrate interactions, and insect social behavior (Guerenstein and Hildebrand, 2008). Sensing $\mathrm{CO}_{2}$ is essential for foraging (Thom et al., 2004), mating (Choi et al., 2018), and oviposition (Myers et al., 1981; Stange, 1997) in many moth species of the Lepidoptera. These studies raised a general question about whether the LPO sensilla in lepidopteran species are sensitive to volatile compounds. Earlier report indicated that the LPO sensilla of Rhodogastria respond to cyclopentanone, acetic acid, octanol, limonene, citral, hexanal, butanal, and pentanal (Bogner et al., 1986), and the LPO sensilla in Pieris brassicae are responding to terpineol, cyclopentanone, cumol, acetic acid, propionic acid, and butyric acid (Bogner, 1990). According to the findings of these two articles, the labial palps in adult Lepidoptera that are excited by stimulation with $\mathrm{CO}_{2}$ may also respond to various volatile compounds. However, it is unknown whether these chemical odors elicited responses of labial palps in other species.

Spodoptera frugiperda (Lepidoptera: Noctuidae), also called fall armyworm, is native to America (Sparks, 1979) but has been spread to Africa (Goergen et al., 2016; Nagoshi et al., 2017; Stokstad, 2017), India (Ganiger et al., 2018), and China (Guo et al., 2018a; Li et al., 2019; Sun et al., 2019a,b). S. frugiperda has a wide host range of more than 350 species of plants, including corn, rice, wheat, soybean, and cotton (Montezano et al., 2018), and is one of the most damaging crop pests. There have been many latest investigations focusing on the management against this pest, such as genome editing of the receptor for Bacillus thuringiensis in S. frugiperda (Jin et al., 2019), the potential roles of Junonia coenia densovirus in S. frugiperda control (Chen et al., 2020), and the positive phototaxis of S. frugiperda (Liu et al., 2020). Elevated $\mathrm{CO}_{2}$ concentration was recently reported to affect the growth and development of S. frugiperda (Zhao et al., 2019), providing support for investigating the structure and function of the $\mathrm{LPO}$, the $\mathrm{CO}_{2}$-sensitive organ. In this study, the distribution and fine structure of sensilla located in the LPO were investigated using scanning electron microscopy. Sensilla in the LPO were divided into two morphological types. Subsequently, we modified the electroantennogram (EAG) setup to function as the electrolabialpalpography (ELPG) to record the responses of labial palps to different concentrations of $\mathrm{CO}_{2}$ and 29 plant volatiles. Finally, the sensilla that responded to $\mathrm{CO}_{2}$ in the LPO were identified via the intracellular recording (ICR). The results indicated that there are two types of sensory neurons in the LPO of $S$. frugiperda, one of which could be strongly activated by different concentrations of $\mathrm{CO}_{2}$, while the other type showed no response to $\mathrm{CO}_{2}$. Our work established a foundation for further study of the roles of labial palps in insect olfaction-related behaviors. Based on these results, further investigations of the function of labial palps and their biological roles together with responses to $\mathrm{CO}_{2}$ and volatile ligands identified in this study of $S$. frugiperda are necessary.

\section{MATERIALS AND METHODS}

\section{Insects Rearing}

The S. frugiperda colony was collected in the wild in Yunnan Province, China, in March, 2019, and then maintained at the Institute of Plant Protection, Chinese Academy of Agricultural Sciences, Beijing, China. The larvae were reared on an artificial diet and placed at $27 \pm 1^{\circ} \mathrm{C}$ with a photoperiod of $14: 10 \mathrm{~h}$ (L:D). Pupae were together kept in a gauze cage before eclosion. Adults were selected by sex and placed in separate test tubes after eclosion and fed 10\% sugar solution every day. Adult females and males were used in all experiments.

\section{Light Microscopy and Biometry Measurements}

The protruding head of adult $S$. frugiperda was fixed to the rim of a pipette tip by using dental wax and observed under a super-high magnification lens zoom 3D microscope (VHX2000, Japan). The labial palps were dissected from the head using fine scissors. Scales covering the labial palps were cleared with double-sided tap. Then, the dehydrated and transparent labial palps were positioned on a microscopic slide with a drop of glycerin and a cover slip. Finally, the labial palps were observed and measured using a super-high magnification lens zoom 3D microscope (VHX-2000, Japan). We measured the 
length of each segment of labial palps, and the depth and diameter of the LPO.

\section{Scanning Electron Microscopy}

The labial palps were removed from 3- to 4-day-old moths and then cleared with double-sided tape to remove the outer scales. In order to study the morphology of the sensilla in LPO, we split the LPO by using fine scissors. Next, these prepared samples were processed by a series of dehydration, drying, and last were sprayed with gold as described by Guo et al. (2018b). In the described steps, the critical point drier was LEICA EM CPD (Germany) and the type of a sputter-coating unit is EIKO IB-3 (Japan). Finally, the samples were investigated using a Hitachi SU8010 scanning electron microscope (Japan) at $10 \mathrm{kV}$.

\section{Electrolabialpalpography}

Taking 3- to 4-day-old adult S. frugiperda, the labial palps were carefully cut from the base charily by using fine scissors, and surface scales were removed with double-sided tape. The treated labial palps were used for recording with the base inserted into the conducting gel (Parker Laboratories, United States) and the tip just contacting the conducting gel to ensure that the opening of the LPO, which harbors all the sensilla, was exposed to the air. The conducting gel was painted on the neutral arms of the metal electrode.

For $\mathrm{CO}_{2}$ stimulus, the mounted labial palp was excited with stimulus delivery in self-regulating stimulus flow controller, which was mainly comprised of a 3/2-way solenoid valve (XP-513, Japan) and two currents of equal flow rate at $0.8 \mathrm{~L} / \mathrm{min}$. One current called continuous flow was diverted through bottled synthetic air, and the other current called stimuli flow was diverted through bottled $\mathrm{CO}_{2}$ at different concentrations. Stimuli were provided for $1 \mathrm{~s}$ by controlling the 3/2-way solenoid valve and were delivered through a $14-\mathrm{cm}$-long metal tube. Commercially available compressed bottled $\mathrm{CO}_{2}$ gas stimuli were used at concentrations of $0.1,1$, and $10 \%$ (the remainder was synthetic air), and synthetic air was used as a control. To make synthetic air $\mathrm{CO}_{2}$-free, it contained only $21 \% \mathrm{O}_{2}$ and $78 \% \mathrm{~N}_{2}$. All above gases in certificated gas cylinders were bought from company (Beijing Shangtonghong Chemical, China). The resulting ELPG amplitudes (negative potential) were recorded and analyzed by using EAG software (Syntech, Germany). The ELPG response values for $\mathrm{CO}_{2}$ were calculated by subtracting the value of the same labial palp corresponding to the blank control (synthetic air).

For odor stimuli, $10 \mu \mathrm{l}$ of test solution or solvent was added in to filter paper strips $(0.5 \mathrm{~cm} \times 6 \mathrm{~cm})$ inserted in a Pasteur pipette (15 cm long). A flow of purified and humidified air continuously blew toward the labial palp through a metal tube at $0.4 \mathrm{~L} / \mathrm{min}$. A stimulus air pulse was added for $200 \mathrm{~ms}$. The intervals between two stimuli were $30 \mathrm{~s}$. The Pasteur pipette connected to the stimulus air controller CS-55 (Syntech, Germany) was used for stimulation. The pre-amplifier was displayed on a computer via a software interface EAGPRO (Syntech, Germany), and action potentials were amplified, digitized, and visualized on a computer screen. The 29 chemical compounds (95\% minimum purity compound) used in this study were purchased from Sigma-Aldrich (Germany). These compounds were dissolved in paraffin oil at the concentration of $1 \mu \mathrm{g} / \mu \mathrm{l}$. For odor stimuli in ELPG assay, the recording of labial palp to paraffin oil was used as a control. The ELPG response values for odorants were calculated by subtracting the value of the same labial palp corresponding to the paraffin oil.

\section{Intracellular Recording}

The 3- to 4-day-old female and male adults after emergence were wedged into a $1 \mathrm{ml}$ plastic pipette tip with the narrow end cut to allow the head and the exposed labial palps to protrude. The protruding head and other organs, including antennae and proboscis, were all immobilized to the edge of the pipette tip with dental wax under a stereomicroscope, just leaving one of the labial palps accessible. The outer scales on the labial palp were removed carefully with double-sided adhesive tape, and then, the labial palp was fixed with dental wax to reveal just the tip of labial palp and the opening of the LPO.

Nerve impulses from single sensory neurons were recorded intracellularly using a sharp quartz electrode. Under a SZX16 microscope (Olympus, Japan), the reference electrode made of a silver wire was inserted into the moth eye, and the recording electrode which containing $0.2 \mathrm{M} \mathrm{KAc}$ was inserted vertically into the LPO via a micromanipulator (Leica, Germany). Spikes were recorded when the quartz electrode was inserted into a sensory neuron in sensilla. During the insertion of the recording electrode into the LPO, it was not possible to distinguish the different sensilla under the microscope because they are located inside the LPO and only the opening of LPO was visible. The amplified analog signals of the action potentials were captured and processed using a signal amplifier (Axoclamp 900A, United States) and a digital-to-analog converter (CED MICRO 1401, England). The recorded spikes activity was displayed on a computer screen using the software package Autospike 28.01 (Syntech, Germany).

For stimulus delivery, a $5 \mathrm{~s} \mathrm{CO}_{2}$ stimulus flow was provided by a self-regulating stimulus flow controller. A flow of purified and humidified synthetic air $\left(21 \% \mathrm{O}_{2}, 78 \% \mathrm{~N}_{2}\right)$ was continuously blown on the opening of the LPO through a 14-cm-long metal tube by the self-regulating stimulus flow controller at $0.8 \mathrm{~L} / \mathrm{min}$. $\mathrm{CO}_{2}$ stimuli were represented at $0.1,1,10 \%$, and synthetic air $\left(21 \% \mathrm{O}_{2}\right.$ and $\left.78 \% \mathrm{~N}_{2}\right)$ was used as a control. The response values to specific concentration of $\mathrm{CO}_{2}$ were calculated using the formula: $\mathrm{T}-\mathrm{C}$, where $\mathrm{T}$ represents the differences in spike numbers observed between $5 \mathrm{~s}$ before and $5 \mathrm{~s}$ after $\mathrm{CO}_{2}$ delivery, and $\mathrm{C}$ represents the differences in spike numbers observed between $5 \mathrm{~s}$ before and $5 \mathrm{~s}$ after control (synthetic air) delivery.

\section{Image Processing and Statistical Analysis}

The classification and naming of sensilla in LPO were described in Zhao et al. (2013). ELPG statistics and graphing were 

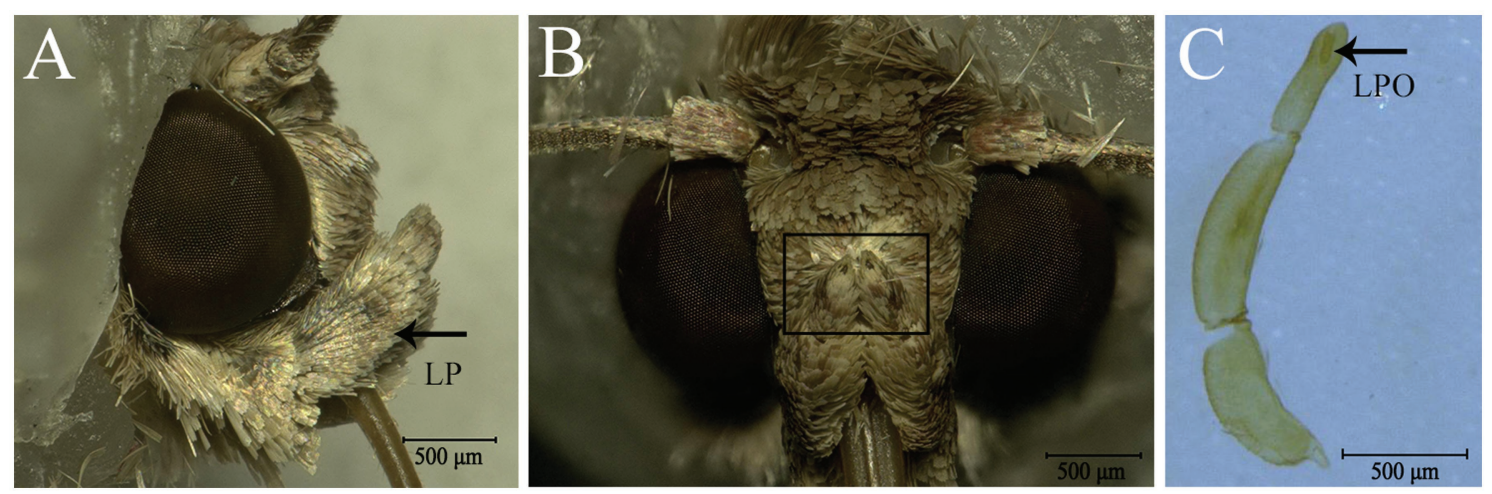

FIGURE 1 | Labial palps (LP) and labial-palp pit organ (LPO) of Spodoptera frugiperda. (A) Lateral view of the head and the right LP (black arrow); the LPs are covered densely with scales and are located on each side of the proboscis just below the eyes. (B) Front view of the head and the LPS. The black boxed area shows the tip of the two LPs and the opening of the LPO. (C) Three segments of the LP; the black arrow shows the LPO.

TABLE 1 | The length of each segment of labial palp (LP) and the depth and diameters of LPO of $S$. frugiperda.

\begin{tabular}{|c|c|c|c|}
\hline & Female & Male & $t$-test \\
\hline $\begin{array}{l}\text { Length of the first LP } \\
\text { segment }(\mu \mathrm{m})\end{array}$ & $688.26 \pm 16.30(23)$ & $663.39 \pm 11.03(23)$ & $p=0.213$ \\
\hline $\begin{array}{l}\text { Length of the second } \\
\text { LP segment }(\mu \mathrm{m})\end{array}$ & $869.57 \pm 17.80(23)$ & $846.04 \pm 11.00(23)$ & $p=0.267$ \\
\hline $\begin{array}{l}\text { Length of the third LP } \\
\text { segment }(\mu \mathrm{m})\end{array}$ & $470.78 \pm 12.04(23)$ & $486.43 \pm 10.27(23)$ & $p=0.328$ \\
\hline Depth of LPO $(\mu \mathrm{m})$ & $118.35 \pm 2.60(17)$ & $116.22 \pm 2.09(23)$ & $p=0.521$ \\
\hline $\begin{array}{l}\text { Diameter of LPO } \\
\text { opening }(\mu \mathrm{m})\end{array}$ & $43.08 \pm 1.43(13)$ & $39.15 \pm 1.03(20)$ & $p=0.029$ \\
\hline $\begin{array}{l}\text { Inner diameter of LPO } \\
\text { at half length }(\mu \mathrm{m})\end{array}$ & $38.77 \pm 1.43(13)$ & $37.53 \pm 0.74(20)$ & $p=0.473$ \\
\hline $\begin{array}{l}\text { Inner diameter of LPO } \\
\text { at the base }(\mu \mathrm{m})\end{array}$ & $33.69 \pm 1.22(13)$ & $32.80 \pm 1.08(20)$ & $p=0.595$ \\
\hline
\end{tabular}

Data in the table are means $\pm S E$. The numbers in parentheses indicate the replicates of measurement.

performed using GraphPad Prism. The measured data of labial palps were analyzed in Microsoft Office Excel 2007. LPO sensilla were measured by LSM Image Browser and analyzed in Microsoft Office Excel 2007. Differences in the response value (or measured data) of females and males were analyzed by $t$-test. Spikes separated from noise were analyzed and evaluated by the computer software Autospike (Syntech, Germany).

\section{RESULTS}

\section{Morphological Structure of the Labial Palp and LPO in Adult S. frugiperda}

Adults of S. frugiperda possess a pair of labial palps located on the ventral side of the head that enfold the proboscis (Figure 1A). The two labial palps are entirely covered by dense scales and have two small holes at the top (Figure 1B). When the scales are removed, each labial palp contains three segments and is tubular (Figure 1C). Each segment of labial palps in S. frugiperda differs in the morphological structure and length (Figure 1C; Table 1). The first segment of the labial palp, which is connected to the head, is about $675 \mu \mathrm{m}$ long, and the second segment is about $857 \mu \mathrm{m}$. The third segment is about $478 \mu \mathrm{m}$ long. An opening near the tip of the third segment extends to a cavity called the LPO (Figures 1C, 2A), which is about $117 \mu \mathrm{m}$ deep and of variable diameter (Table 1). In females, the diameter of the LPO opening is $43.08 \pm 1.43 \mu \mathrm{m}$ (mean $\pm \mathrm{SE}, n=13$ ). In males, the diameter of the LPO opening is $39.15 \pm 1.03 \mu \mathrm{m}$ (mean \pm SE, $n=20$ ). The diameter of the LPO opening in females is significantly longer than in males. The inner diameter of the LPO at the midpoint is approximately $38 \mu \mathrm{m}$, and the inner diameter of the LPO at the base is about $33 \mu \mathrm{m}$.

\section{Fine Morphological Structure of Sensilla Located in LPO}

The LPO is densely packed with approximately 300 sensilla, which comprise hair-shaped sensilla and club-shaped sensilla (Figure 2C). The hair-shaped sensilla are slender, and the ends are slightly bent (Figures 2B,D,E). Some hair-shaped sensilla have forked tips (Figure 2B; blue arrow). The club-shaped sensilla are short and rod-like, and their surfaces have grooves (Figures 2D,F). Hair-shaped sensilla and club-shaped sensilla are distributed in separate areas along the vertical axis of the LPO (Figure 2D). The length and basal diameter of each sensillum category are shown in Table 2 . In females, the hairshaped sensilla are $23.92 \pm 0.58 \mu \mathrm{m}$ long (mean $\pm \mathrm{SE}, n=5$ ) and the basal diameters are $2.74 \pm 0.25 \mu \mathrm{m}$ (mean $\pm \mathrm{SE}$, $n=5$ ), while the club-shaped sensilla are $13.10 \pm 0.54 \mu \mathrm{m}$ long (mean \pm SE, $n=5$ ) and the basal diameters are $2.02 \pm 0.06 \mu \mathrm{m}$ long (mean $\pm \mathrm{SE}, n=5$ ). In males, the hairshaped sensilla are $25.44 \pm 0.50 \mu \mathrm{m}$ long (mean \pm SE, $n=14$ ) and the basal diameters are $2.88 \pm 0.15 \mu \mathrm{m}$ (mean $\pm \mathrm{SE}$, $n=4$ ), while the club-shaped sensilla are $12.80 \pm 0.27 \mu \mathrm{m}$ long (mean $\pm \mathrm{SE}, n=14$ ) and the basal diameters are $2.08 \pm 0.03 \mu \mathrm{m}$ (mean $\pm \mathrm{SE}, n=14$ ). The $t$-test results show 

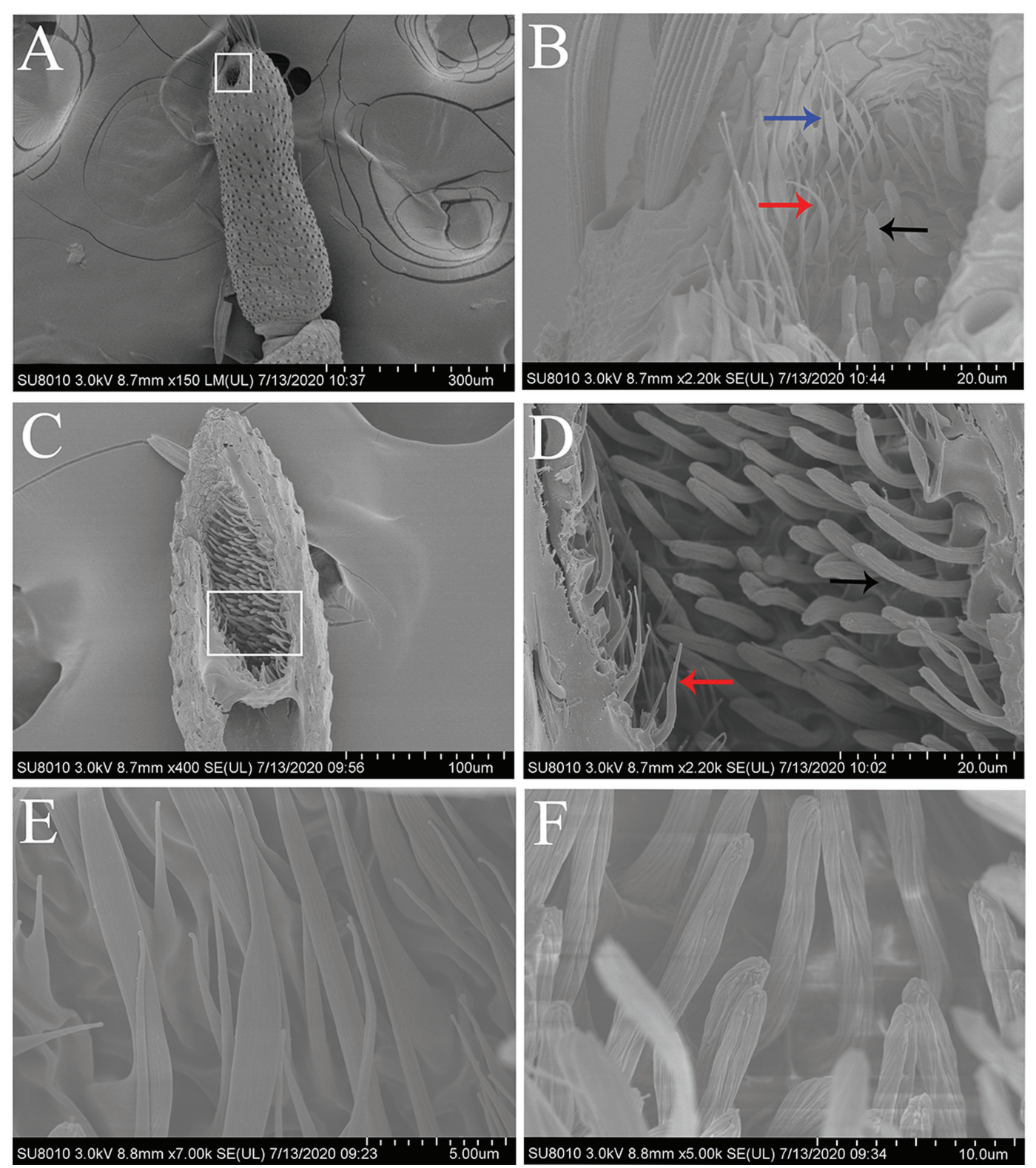

FIGURE 2 | Scanning electron micrographs of the terminal segment of labial palps and the LPO sensilla in S. frugiperda. (A) The terminal segment of the labial palp showing the opening of the LPO. White boxed area: opening of the LPO. (B) Scanning electron micrograph of the sensilla located around the opening of the LPO, which contains hair-shaped sensilla (red and blue arrows) and club-shaped sensilla (black arrow). Two hair-shaped sensilla subtypes were found hair-shaped sensilla (red arrow) and hair-shaped sensilla with forked tips (blue arrow). (C) Longitudinal section of the LPO in one labial palp showing two main types of sensilla.

(D) Enlarged image of white boxed area in (C). The LPO has two types of sensilla, i.e., hair-shaped sensilla (red arrow) and club-shaped sensilla (black arrow). These two types of sensilla are distributed in separate areas along the vertical axis of the LPO. (E) Scanning electron micrograph of hair-shaped sensilla. (F) Scanning electron micrograph of club-shaped sensilla.

no significant difference in the size of each sensillum type between females and males (Table 2).

\section{ELPG Response of Labial Palp to $\mathrm{CO}_{2}$ and Plant Volatiles}

In order to demonstrate the labial palps of $S$. frugiperda also response to odor stimulation besides $\mathrm{CO}_{2}$ stimulation, we performed ELPG on the female and male labial palps (Figure 3A). The labial palp in S. frugiperda adults responded obviously to different concentrations of $\mathrm{CO}_{2}$. The magnitude of response mainly depended on the concentration of $\mathrm{CO}_{2}$, with the strongest responses to stimulus of $1 \% \mathrm{CO}_{2}$, at about $0.26 \pm 0.02 \mathrm{mV}$ (mean $\pm \mathrm{SE}, n=30$ ), and the weakest responses to the stimulus of $0.1 \% \quad \mathrm{CO}_{2}$, at about $0.18 \pm 0.02 \mathrm{mV}$ (mean $\pm \mathrm{SE}, n=30$; Figure 3B). In females, 
TABLE 2 | The length of LPO sensilla in S. frugiperda and their diameter at the base.

\begin{tabular}{lccc}
\hline & \multicolumn{1}{c}{ Female } & Male & $\boldsymbol{t}$-test \\
\hline $\begin{array}{l}\text { Length of hair-shaped } \\
\text { sensilla }(\mu \mathrm{m})\end{array}$ & $23.92 \pm 0.58(5)$ & $25.44 \pm 0.50(14)$ & $p=0.058$ \\
$\begin{array}{l}\text { Basal diameter of } \\
\text { hair-shaped sensilla }(\mu \mathrm{m})\end{array}$ & $2.74 \pm 0.11(5)$ & $2.87 \pm 0.15(14)$ & $p=0.48$ \\
$\begin{array}{l}\text { Length of club-shaped } \\
\text { sensilla }(\mu \mathrm{m})\end{array}$ & $13.10 \pm 0.54(5)$ & $12.79 \pm 0.27(14)$ & $p=0.46$ \\
$\begin{array}{l}\text { Basal diameter of } \\
\text { club-shaped sensilla }\end{array}$ & $2.02 \pm 0.06(5)$ & $2.07 \pm 0.03(14)$ & $p=0.37$ \\
\hline
\end{tabular}

Data in the table are means \pm SE. The numbers in parentheses indicate the replicates of measurement.

the responses of labial palp to $1 \% \mathrm{CO}_{2}$ are significantly greater than to $0.1 \% \mathrm{CO}_{2}$. There is no significant difference in the responses of labial palp to $1 \% \mathrm{CO}_{2}$ and $10 \% \mathrm{CO}_{2}$, and $0.1 \% \mathrm{CO}_{2}$ and $10 \% \mathrm{CO}_{2}$. In males, the responses of labial palp to $1 \% \mathrm{CO}_{2}$ is significantly greater than to $0.1 \%$ $\mathrm{CO}_{2}$ and is significantly less than to $10 \% \mathrm{CO}_{2}$. There is no significant difference in the responses of labial palp to $0.1 \%$ $\mathrm{CO}_{2}$ and $10 \% \mathrm{CO}_{2}$ (Figure 3B). However, The response value of labial palp to the same concentration of $\mathrm{CO}_{2}$ was not significantly different between females and males (Figure 3B).

To verify whether the labial palp, as an olfactory organ, responded to volatile compounds other than $\mathrm{CO}_{2}$, we also investigated the electrophysiological responses of labial palps to 29 volatile compounds (Figure 3C). The labial palps of S. frugiperda obviously responded to six compounds: butylamine, heptylamine, heptanal, valeraldehyde, propionic acid, and acetic acid (Figure 3C). As with $\mathrm{CO}_{2}$, responses were not significantly different between females and males.

\section{Recording From LPO Sensilla to $\mathrm{CO}_{2}$}

In order to check the existence of sensilla in the LPO that respond to $\mathrm{CO}_{2}$, we performed ICR on sensory neurons in LPO sensilla from male and female labial palps. $\mathrm{CO}_{2}{ }^{-}$ sensitive neurons were found in LPO sensilla of adult S. frugiperda (Figure 4; $\mathrm{SN}-\mathrm{a}$ ). We also found sensory neurons that did not respond to $\mathrm{CO}_{2}$ in the LPO sensilla (Figure 4; SN-b). The sensory neurons that responded to $\mathrm{CO}_{2}$ were labeled sensory neuron a $(\mathrm{SN}-\mathrm{a})$, while those that did not respond to $\mathrm{CO}_{2}$ were labeled sensory neuron b (SN-b; Figure 4). We successfully recorded 11 adults S. frugiperda in total, including six females and five males. A total of 22 neurons with unambiguous spikes from eight insects (four females and four males) were analyzed. For $\mathrm{SN}-\mathrm{a}$, there was a strong excitatory response to $\mathrm{CO}_{2}$ stimulus at concentrations of $0.1,1$, and $10 \%$ (Figure $4 \mathbf{A}$ ) and the mean activated spikes of these neurons were, respectively, about 93 spikes/5 s, 107 spikes/5 s, and 99 spikes/5 s (Figure 4B). Besides, the responses of $\mathrm{SN}-\mathrm{a}$ were not significantly different between these three concentrations of $\mathrm{CO}_{2}$.

\section{DISCUSSION}

Structure characterization of an olfactory organ and its sensilla are vital to understand how the olfactory organ performs its ecological function. This model has been widely used in the surveys of antennae in lepidopteran insects. In an effort to research the function of another crucial olfactory organ, the labial palp, the fine structure of LPO and LPO sensilla in $S$. frugiperda were investigated in detailed in the present study. For the structure of LPO, we found a significant difference in the diameter of the LPO opening between females and males in $S$. frugiperda. The diameter of the LPO opening in S. frugiperda exhibited distinct sexual dimorphism and was much longer in females $(43.08 \mu \mathrm{m})$ than in males $(39.15 \mu \mathrm{m})$. In other reported Noctuidae species (Zhao et al., 2013; Dong et al., 2014), the diameter of the LPO opening tends to be the same size in both sexes. The sexual dimorphism of the diameter of the LPO opening is described for the first time in this study, although sexual dimorphism also occurs in the length of labial palps in Cactoblastis cactorum (Stange et al., 1995), Mythimna separata (Dong et al., 2014), Carposina sasakii (Chen and Hua, 2016), and Plutella xylostella (Yan et al., 2019). This phenomenon may be related to sex-specific differences in behavior, such as courtship and oviposition. For example, C. cactorum probes the surface of a plant with their labial palps before ovipositing, so the length of labial palps in females is much longer than in males (Stange et al., 1995). The differences between the sexes in the diameter of the LPO opening may also be due to the ovipositing behavior of female $S$. frugiperda, though further studies are required to confirm this.

In our study, the densely packed array of LPO sensilla in $S$. frugiperda can be divided into two morphological types: hair-shaped sensilla and club-shaped sensilla, like that in C. cactorum (Stange et al., 1995), Helicoverpa armigera (Zhao et al., 2013), M. separata (Dong et al., 2014), and C. sasakii (Chen and Hua, 2016); hair-shaped sensilla in C. cactorum and C. sasakii have not been described in detail. However, there is only one kind of LPO sensilla in some moth species. For example, in Rhodogastria spp. the LPO is densely packed with smooth-walled sensilla of uniform appearance (Bogner et al., 1986), and the LPO in Plodia interpunctella also contains a single small trichoid sensillum (Barcaba and Krenn, 2015). The LPO in Grapholita molesta (Song et al., 2016) contains three categories of sensilla: hairshaped sensilla, club-shaped sensilla, and small mastoid sensilla. Although the categories of LPO sensilla are identical, the hair-shaped sensilla and club-shaped sensilla in the LPO of $S$. frugiperda are distributed in separate areas along the vertical axis of LPO, whereas they are situated in the upper half and the lower half of the pit in H. armigera (Zhao et al., 2013) and $M$. separata (Dong et al., 2014). This type of distribution of LPO sensilla in S. frugiperda is described for the first time. In summary, the differences of LPO sensilla in categories and location may be dependent on the insect species, or related to the behavior of insects and the function of the labial palps. 
A

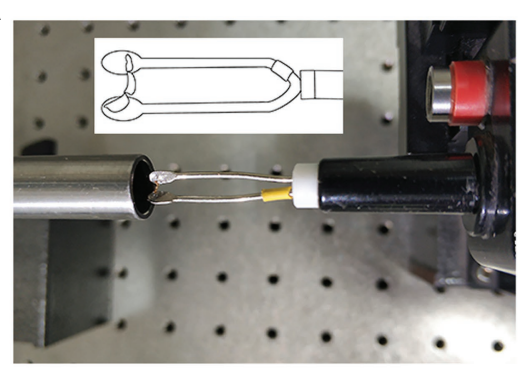

B

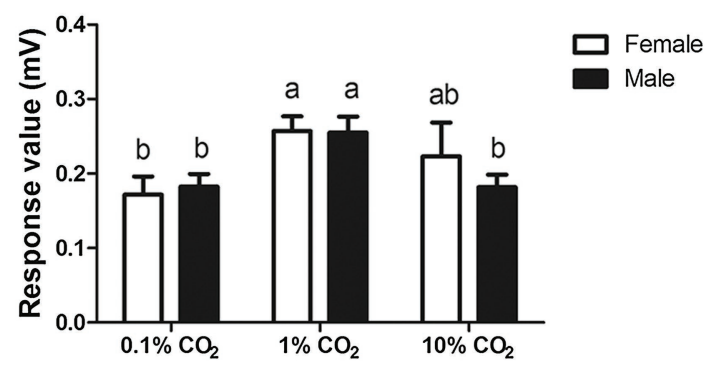

C

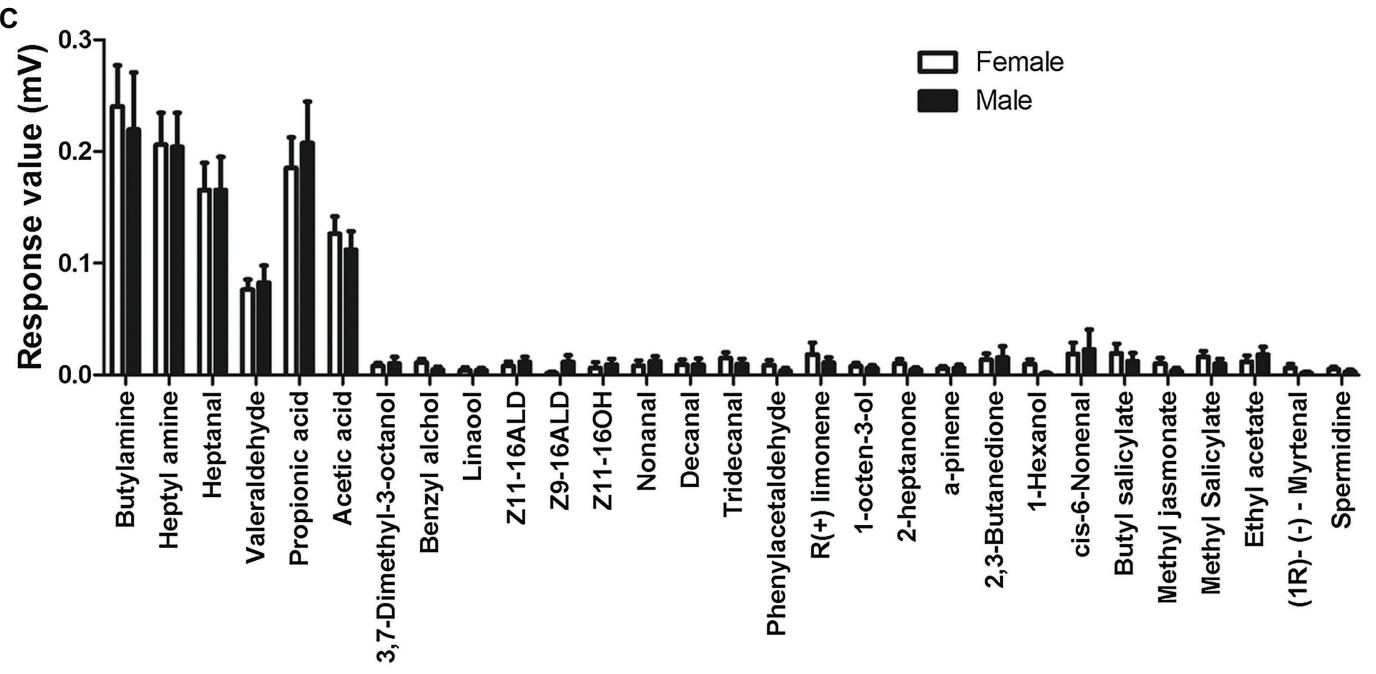

FIGURE 3 | Electrolabialpalpography (ELPG) response of labial palps in S. frugiperda to $\mathrm{CO}_{2}$ and plant volatiles. (A) Schematic showing a labial palp mounted on the electrode in the ELPG assay. (B) ELPG response of labial palp in S. frugiperda to different concentrations of $\mathrm{CO}_{2}$ including $0.1,1$, and $10 \%$. Data are the mean \pm SD $(n=30)$. (C) ELPG responses of labial palp in S. frugiperda to 29 plant volatiles. Data are the mean \pm SE $(n=15)$.

Electrolabialpalpography and ICR data in the present investigation support the idea that $S$. frugiperda have $\mathrm{CO}_{2}$ sensitive neurons in the LPO, as reported in other lepidopteran species (Bogner et al., 1986; Stange et al., 1995; Stange, 1997; Guerenstein et al., 2004; Ning et al., 2016). This suggests that $\mathrm{CO}_{2}$-detection is a universal function of the LPO in Lepidoptera. Interestingly, we found a kind of sensory neuron that was non-responsive to $\mathrm{CO}_{2}$ in the LPO of $S$. frugiperda, which has never been reported before. This finding implies that LPO sensilla are not uniform in detecting $\mathrm{CO}_{2}$ and they may also respond to other odorants. Our electrophysiological recording results strongly support the hypothesis that LPO sensilla can respond to volatile chemicals. The labial palps of S. frugiperda obviously responded to six of 29 volatiles tested in our experiment, including butylamine, heptylamine, heptanal, valeraldehyde, propionic acid, and acetic acid. Electrophysiologically active compounds in this study, propionic acid and acetic acid, which are volatiles from host plants, have been reported in other lepidopteran insects (Bogner et al., 1986; Bogner, 1990). In addition, several kinds of odorants and their analogues found to be effective stimuli in S. frugiperda also activate $\mathrm{CO}_{2}$ receptors of antennae in flies and $\mathrm{CO}_{2}$ receptors of maxillary palps in mosquitoes
(Turner and Ray, 2009; Tauxe et al., 2013; Macwilliam et al., 2018). The class of odorants also present in ripe fruits has important ecological significance, as they can modify the $\mathrm{CO}_{2}$ avoidance behavior, helping the host-seeking behavior of Drosophila melanogaster (Turner and Ray, 2009). Hence, we predict that olfactory perception of ecologically relevant volatiles occurs on labial palps of $S$. frugiperda, but its role in behaviors remains to be investigated.

We speculate that the gustatory receptor (GR) genes and ionotropic receptor (IR) genes have pivotal roles in detecting $\mathrm{CO}_{2}$ and other volatile compounds for $S$. frugiperda LPO. Two GRs, GR21a and GR63a, were identified as the $\mathrm{CO}_{2}$ receptor genes for the first time in $D$. melanogaster (Jones et al., 2007; Kwon et al., 2007). Later, their homologous genes, GR1, GR2, and GR3, were successively identified as the $\mathrm{CO}_{2}$ receptors in many mosquito species (Kent et al., 2008; Robertson and Kent, 2009; Coutinho-Abreu et al., 2019). GR1, GR2, and GR3, which are highly expressed in labial palps, have been identified using phylogenetic analysis in several lepidopteran species (Briscoe, 2000; Spaethe and Briscoe, 2004; Liu et al., 2014; Xu and Anderson, 2015; Zhang et al., 2015; Liu et al., 2017), and their functions have been confirmed (Xu and Anderson, 2015; Ning et al., 2016). 


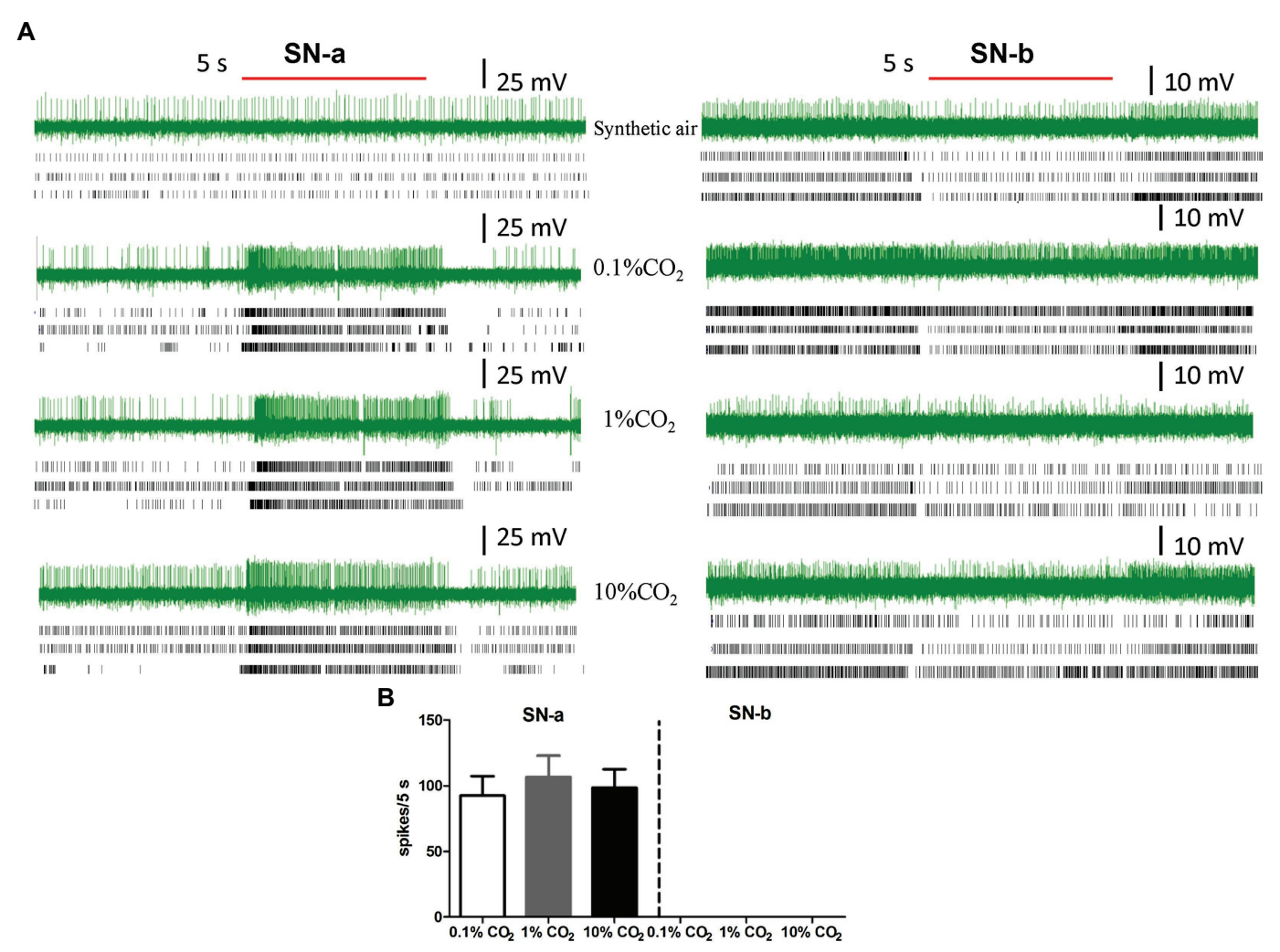

FIGURE 4 | Intracellular recording (ICR) analysis of the sensory neurons housed in LPO sensilla. (A) The representative traces of ICRs, three example of rater plots of action potentials of responses of sensory neurons in $\mathrm{SN}-\mathrm{a}$ and $\mathrm{SN}-\mathrm{b}$ to synthetic air, $0.1 \% \mathrm{CO}_{2}, 1 \% \mathrm{CO}_{2}$, and $10 \% \mathrm{CO}_{2}$. The red bold line represents the $5 \mathrm{~s}$ stimulation. The letters (SN-a, SN-b) represent the different types of sensory neurons in LPO sensilla. (B) Quantification of the mean responses of $\mathrm{SN}-\mathrm{a}$ and $\mathrm{SN}-\mathrm{b}$ in $\mathrm{LPO}$ sensilla to $0.1 \% \mathrm{CO}_{2}, 1 \% \mathrm{CO}_{2}$, and $10 \% \mathrm{CO}_{2}$. Data are the mean $\pm \mathrm{SE}(n=10-16)$. The response values to specific concentration of $\mathrm{CO}_{2}$ were calculated using the formula: $\mathrm{T}-\mathrm{C}$, where T represents the differences in spike numbers observed between $5 \mathrm{~s}$ before and $5 \mathrm{~s}$ after $\mathrm{CO}_{2}$ delivery, and $\mathrm{C}$ represents the differences in spike numbers observed between $5 \mathrm{~s}$ before and $5 \mathrm{~s}$ after control (synthetic air) delivery.

These three GRs are likely required for $\mathrm{CO}_{2}$ detection in S. frugiperda. For volatile compounds detection, the molecular mechanism is generally related to odorant receptors (ORs). However, these six odors, which excited labial palps of S. frugiperda, mainly contain acid, aldehyde, and amine. It has been reported that sensing of this class of odors was involved in IRs predominantly (Zhang and Wang, 2020). Moreover, there were indeed IRs identified in labial palps of the lepidopteran $H$. armigera (Guo et al., 2018b). Analogously, IRs might also be the receptor for detecting these six odors in S. frugiperda.

Our exploration of ultrastructural characteristics of LPO sensilla and their physiological functions in S. frugiperda might be useful not only for obtaining knowledge about the function of labial palps but also for controlling this serious insect pest. Further study is needed to clarify the physiological functions of the two morphological types of sensilla in LPO, hair-shaped sensilla and club-shaped sensilla, and confirm that these two types of LPO sensilla in S. frugiperda are separately sensitive to $\mathrm{CO}_{2}$ and airborne chemicals. Further behavioral studies and molecular investigations of the labial palps are necessary to better understand the ecological significance and molecular basis of olfaction in S. frugiperda.

\section{DATA AVAILABILITY STATEMENT}

The original contributions presented in the study are included in the article/supplementary material, and further inquiries can be directed to the corresponding author.

\section{AUTHOR CONTRIBUTIONS}

JS and GW designed the experiments. QC, XL, SC, BM, and MG performed the experiments. QC and GW wrote the manuscript and analyzed the data. JS and GW revised the manuscript. All authors contributed to the article and approved the submitted version.

\section{FUNDING}

This work was supported by the National Natural Science Foundation of China (31861133019 and 31725023). 


\section{REFERENCES}

Barcaba, T., and Krenn, H. W. (2015). The mouthparts of adult Indian meal moths, Plodia interpunctella (Hübner, 1813) (Lepidoptera: Pyralidae). Entomol. Aust. 22, 91-105.

Bogner, F. (1990). Sensory physiological investigation of carbon dioxide receptors in Lepidoptera. J. Insect Physiol. 36, 951-957. doi: 10.1016/0022-1910(90) 90083-R

Bogner, F., Boppré, M., Ernst, K. D., and Boeckh, J. (1986). CO $\mathrm{CO}_{2}$ sensitive receptors on labial palps of Rhodogastria moths (Lepidoptera: Arctiidae): physiology, fine structure and central projection. J. Comp. Physiol. A 158, 741-749. doi: $10.1007 / \mathrm{BF} 01324818$

Bohbot, J. D., Sparks, J. T., and Dickens, J. C. (2014). The maxillary palp of Aedes aegypti, a model of multisensory integration. Insect Biochem. Mol. Biol. 48, 29-39. doi: 10.1016/j.ibmb.2014.02.007

Briscoe, A. D. (2000). Six opsins from the butterfly Papilio glaucus: molecular phylogenetic evidence for paralogous origins of red-sensitive visual pigments in insects. J. Mol. Evol. 51, 110-121. doi: 10.1007/s002390010071

Chen, J., and Hua, B. (2016). Sexual dimorphism of adult labial palps of the peach fruit moth Carposina sasakii Matsumura (Lepidoptera: Carposinidae) with notes on their sensilla. Acta Zool. 97, 42-48. doi: 10.1111/azo.12103

Chen, Z. W., Yang, Y. C., Zhang, J. F., Jin, M. H., Xiao, Y. T., Xia, Z. C., et al. (2020). Susceptibility and tissue specificity of Spodoptera frugiperda to Junonia coenia densovirus. J. Integr. Agr. 20, 840-849. doi: 10.1016/ S2095-3119(20)63163-X

Choi, K. S., Ahn, S. J., Kim, S. B., Ahn, J. J., Jung, B. N., Go, S. W., et al. (2018). Elevated $\mathrm{CO}_{2}$ may alter pheromonal communication in Helicoverpa armigera (Lepidoptera: Noctuidae). Physiol. Entomol. 43, 169-179. doi: 10.1111/ phen.12239

Coutinho-Abreu, L. V., Sharma, K., Cui, L., Yan, G., and Ray, A. (2019). Odorant ligands for the $\mathrm{CO}_{2}$ receptor in two Anopheles vectors of malaria. Sci. Rep. 9:2549. doi: 10.1038/s41598-019-39099-0

Dong, J., Liu, H., Tang, Q., Liu, Y., Zhao, X., and Wang, G. (2014). Morphology, type and distribution of the labial-palp pit organ and its sensilla in the oriental armyworm, Mythimna separata (Lepidoptera: Noctuidae). Acta Entomol. Sin. 57, 681-687. doi: 10.16380/j.kcxb.2014.06.012

Faucheux, M. J. (2008). Mouthparts and associated sensilla of a South American moth Synempora andesae (Lepidoptera: Neopseustidae). Rev. Soc. Entomol. Argent. 67, 21-33. doi: 10.1590/S0373-56802008000100003

Galizia, C. G., and Rossler, W. (2010). Parallel olfactory systems in insects: anatomy and function. Annu. Rev. Entomol. 55, 399-420. doi: 10.1146/ annurev-ento-112408-085442

Ganiger, P. C., Yeshwanth, H. M., Muralimohan, K., Vinay, N., Kumar, A. R. V., and Chandrashekara, K. (2018). Occurrence of the new invasive pest, fall armyworm, Spodoptera frugiperda (JE Smith) (Lepidoptera: Noctuidae), in the maize fields of Karnataka, India. Curr. Sci. 115, 621-623. doi: 10.18520/ cs/v115/i4/621-623

Goergen, G., Kumar, P. L., Sankung, S. B., Togola, A., and Tamo, M. (2016). First report of outbreaks of the fall armyworm Spodoptera frugiperda (J E Smith) (Lepidoptera, Noctuidae), a new alien invasive pest in west and central Africa. PLoS One 11:e0165632. doi: 10.1371/journal.pone.0165632

Guerenstein, P. G., Christensen, T. A., and Hildebrand, J. G. (2004). Sensory processing of ambient $\mathrm{CO}_{2}$ information in the brain of the moth Manduca sexta. J. Comp. Physiol. A 190, 707-725. doi: 10.1007/s00359-004-0529-0

Guerenstein, P. G., and Hildebrand, J. G. (2008). Roles and effects of environmental carbon dioxide in insect life. Annu. Rev. Entomol. 53, 161-178. doi: 10.1146/ annurev.ento.53.103106.093402

Guo, J. F., Zhao, J. Z., He, K. L., Zhang, F., and Wang, Z. Y. (2018a). Potential invasion of the crop-devastating insect pest fall armyworm Spodoptera frugiperda to China. Plant Prot. 44, 1-10. doi: 10.16688/j.zwbh.2018452

Guo, M. B., Chen, Q. Y., Liu, Y., Wang, G. R., and Han, Z. J. (2018b). Chemoreception of mouthparts: sensilla morphology and discovery of chemosensory genes in proboscis and labial palps of adult Helicoverpa armigera (Lepidoptera: Noctuidae). Front. Physiol. 9:970. doi: 10.3389/ fphys.2018.00970

Jin, M. H., Tao, J. H., Li, Q., Cheng, Y., Sun, X. X., Wu, K. M., et al. (2019). Genome editing of the SfABCC2 gene confers resistance to Cry1F toxin from Bacillus thuringiensis in Spodoptera frugiperda. J. Integr. Agr. 20, 815-820. doi: 10.1016/S2095-3119(19)62772-3
Jones, W. D., Cayirlioglu, P., Kadow, I. G., and Vosshall, L. B. (2007). Two chemosensory receptors together mediate carbon dioxide detection in Drosophila. Nature 445, 86-90. doi: 10.1038/nature05466

Kent, K. S., Harrow, I. D., Quartararo, P., and Hildebrand, J. G. (1986). An accessory olfactory pathway in Lepidoptera: the labial pit organ and its central projections in Manduca sexta and certain other sphinx moths and silk moths. Cell Tissue Res. 245, 237-245. doi: 10.1007/BF00213927

Kent, L. B., Walden, K. K., and Robertson, H. M. (2008). The Gr family of candidate gustatory and olfactory receptors in the yellow-fever mosquito Aedes aegypti. Chem. Senses 33, 79-93. doi: 10.1093/chemse/bjm067

Kwon, J. Y., Dahanukar, A., Weiss, L. A., and Carlson, J. R. (2007). The molecular basis of $\mathrm{CO}_{2}$ reception in Drosophila. Proc. Natl. Acad. Sci. 104, 3574-3578. doi: 10.1073/pnas.0700079104

Lee, J. K., Selzer, R., and Altner, H. (1985). Lamellated outer dendritic segments of a chemoreceptor within wall-pore sensilla in the labial palp-pit organ of the butterfly, Pieris rapae L. (Insecta, Lepidoptera). Cell Tissue Res. 240, 333-342. doi: 10.1007/BF00222343

Li, G. P., Ji, T. J., Sun, X. X., Jiang, Y. Y., Wu, K. M., and Feng, H. Q. (2019). Susceptibility evaluation of invaded Spodoptera frugiperda population in Yunnan province to five Bt toxins. Plant Prot. 45, 15-20. doi: 10.16688/j. zwbh.2019201

Liu, N. Y., Xu, W., Papanicolaou, A., Dong, S. L., and Anderson, A. (2014). Identification and characterization of three chemosensory receptor families in the cotton bollworm Helicoverpa armigera. BMC Genomics 15:597. doi: 10.1186/1471-2164-15-597

Liu, Y. J., Zhang, D. D., Yang, L. Y., Dong, Y. H., Liang, G. M., Donkersley, P., et al. (2020). Analysis of phototactic responses in Spodoptera frugiperda using Helicoverpa armigera as control. J. Integr. Agr. 20, 821-828. doi: 10.1016/ S2095-3119(19)62863-7

Liu, Z., Wang, X., Lei, C., and Zhu, F. (2017). Sensory genes identification with head transcriptome of the migratory armyworm Mythimna separata. Sci. Rep. 7:46033. doi: 10.1038/srep46033

Macwilliam, D., Kowalewski, J., Kumar, A., Pontrello, C., and Ray, A. (2018). Signaling mode of the broad-spectrum conserved $\mathrm{CO}_{2}$ receptor is one of the important determinants of odor valence in Drosophila. Neuron 97, 1153. e4-1167.e4. doi: 10.1016/j.neuron.2018.01.028

Montezano, D. G., Specht, A., Sosa-Gómez, D. R., Roque-Specht, V. F., Sousa-Silva, J. C., Paula-Moraes, S. V., et al. (2018). Host plants of Spodoptera frugiperda (Lepidoptera: Noctuidae) in the Americas. Afr. Entomol. 26, 286-300. doi: 10.4001/003.026.0286

Myers, J. H., Monro, J., and Murray, N. (1981). Egg clumping, host plant selection and population regulation in Cactoblastis cactorum (Lepidoptera). Oecologia 51, 7-13. doi: 10.1007/BF00344644

Nagoshi, R. N., Koffi, D., Agboka, K., Tounou, K. A., Banerjee, R., Jurat-Fuentes, J. L., et al. (2017). Comparative molecular analyses of invasive fall armyworm in Togo reveal strong similarities to populations from the eastern United States and the Greater Antilles. PLoS One 12:e0181982. doi: 10.1371/journal.pone.0181982

Ning, C., Yang, K., Xu, M., Huang, L. Q., and Wang, C. Z. (2016). Functional validation of the carbon dioxide receptor in labial palps of Helicoverpa armigera moths. Insect Biochem. Mol. Biol. 73, 12-19. doi: 10.1016/j. ibmb.2016.04.002

Robertson, H. M., and Kent, L. B. (2009). Evolution of the gene lineage encoding the carbon dioxide receptor in insects. J. Insect Sci. 9:19. doi: 10.1673/031.009.1901

Song, Y. Q., Sun, H. Z., and Wu, J. X. (2016). Ultrastructural characteristics of the proboscis and the labial palp pit organ in the oriental fruit moth, Grapholita molesta. Bull. Insectol. 69, 59-66.

Spaethe, J., and Briscoe, A. D. (2004). Early duplication and functional diversification of the opsin gene family in insects. Mol. Biol. Evol. 21, 1583-1594. doi: 10.1093/molbev/msh162

Sparks, A. N. (1979). A review of the biology of the fall armyworm. Fla. Entomol. 62, 82-87. doi: 10.2307/3494083

Stange, G. (1997). Effects of changes in atmospheric carbon dioxide on the location of hosts by the moth, Cactoblastis cactorum. Oecologia 110, 539-545. doi: $10.1007 / \mathrm{s} 004420050192$

Stange, G., Monro, J., Stowe, S., and Osmond, C. B. (1995). The $\mathrm{CO}_{2}$ sense of the moth Cactoblastis cactorum and its probable role in the biological control of the CAM plant Opuntia stricta. Oecologia 102, 341-352. doi: $10.1007 / \mathrm{BF} 00329801$ 
Stange, G., and Stowe, S. (1999). Carbon-dioxide sensing structures in terrestrial arthropods. Microsc. Res. Tech. 47, 416-427. doi: 10.1002/(SICI)1097-0029 (19991215)47:6<416::AID-JEMT5>3.0.CO;2-X

Stokstad, E. (2017). New crop pest takes Africa at lightning speed. Science 356, 473-474. doi: 10.1126/science.356.6337.473

Sun, X. X., Hu, C. X., Jia, H. R., Wu, Q. L., Shen, X. J., Zhao, S. Y., et al. (2019a). Case study on the first immigration of fall armyworm Spodoptera frugiperda invading into China. J. Integr. Agr. 20, 664-672. doi: 10.1016/S2095-3119(19)62839-X

Sun, X. X., Zhao, S. Y., Jin, M. H., Zhao, H. Y., Li, G. P., Zhang, H. W., et al. (2019b). Larval spatial distribution pattern and sampling technique of the fall armyworm Spodoptera frugiperda in maize fields. Plant Prot. 45, 13-18. doi: $10.16688 /$ j.zwbh.2019115

Syed, Z., and Leal, W. S. (2007). Maxillary palps are broad spectrum odorant detectors in Culex quinquefasciatus. Chem. Senses 32, 727-738. doi: 10.1093/ chemse/bjm040

Tauxe, G. M., Macwilliam, D., Boyle, S. M., Guda, T., and Ray, A. (2013). Targeting a dual detector of skin and $\mathrm{CO}_{2}$ to modify mosquito host seeking. Cell 155, 1365-1379. doi: 10.1016/j.cell.2013.11.013

Thom, C., Guerenstein, P. G., Mechaber, W. L., and Hildebrand, J. G. (2004). Floral $\mathrm{CO}_{2}$ reveals flower profitability to moths. J. Chem. Ecol. 30, 1285-1288. doi: 10.1023/B:JOEC.0000030298.77377.7d

Turner, S. L., and Ray, A. (2009). Modification of $\mathrm{CO}_{2}$ avoidance behaviour in Drosophila by inhibitory odorants. Nature 461, 277-281. doi: 10.1038/nature08295

$\mathrm{Xu}, \mathrm{W}$., and Anderson, A. (2015). Carbon dioxide receptor genes in cotton bollworm Helicoverpa armigera. Sci. Nat. 102:11. doi: 10.1007/s00114-015-1260-0

Yan, X. Z., Wang, Z. Y., Duan, Y., Feng, X. M., Deng, C. P., Wu, A. H., et al. (2019). Ultrastructure of sensilla on labial palps and the central projection of their sensory neurons in Plutella xylostella (Lepidoptera: Plutellidae) adults. Acta Entomol. Sin. 62, 205-214. doi: 10.16380/j.kcxb.2019.02.007

Zhang, J., Wang, B., Dong, S., Cao, D., Dong, J., Walker, W. B., et al. (2015). Antennal transcriptome analysis and comparison of chemosensory gene families in two closely related noctuidae moths, Helicoverpa armigera and H. assulta. PLoS One 10:e117054. doi: 10.1371/journal.pone.0117054

Zhang, X. X., and Wang, G. R. (2020). Advances in research on the identification and function of ionotropic receptors in insects. Chinese J. Appl. Entomol. 57, 1046-1055. doi: 10.7679/j.issn.2095-1353.2020.105

Zhao, W. J., He, S. Q., Lu, Z. H., Liu, M. R., Ma, L. Q., Wang, J., et al. (2019). Direct effects of elevated $\mathrm{CO}_{2}$ concentration on development of fall armyworm Spodoptera frugiperda (J. E. Smith). J. Environ. Ent. 41, 736-741. doi: 10.3969/j. issn.1674-0858.2019.04.7

Zhao, X. C., Tang, Q. B., Berg, B. G., Liu, Y., Wang, Y. R., Yan, F. M., et al. (2013). Fine structure and primary sensory projections of sensilla located in the labial-palp pit organ of Helicoverpa armigera (Insecta). Cell Tissue Res. 282, 237-249. doi: 10.1007/BF00319115

Conflict of Interest: The authors declare that the research was conducted in the absence of any commercial or financial relationships that could be construed as a potential conflict of interest.

Publisher's Note: All claims expressed in this article are solely those of the authors and do not necessarily represent those of their affiliated organizations, or those of the publisher, the editors and the reviewers. Any product that may be evaluated in this article, or claim that may be made by its manufacturer, is not guaranteed or endorsed by the publisher.

Copyright (c) 2021 Chen, Liu, Cao, Ma, Guo, Shen and Wang. This is an openaccess article distributed under the terms of the Creative Commons Attribution License (CC BY). The use, distribution or reproduction in other forums is permitted, provided the original author(s) and the copyright owner(s) are credited and that the original publication in this journal is cited, in accordance with accepted academic practice. No use, distribution or reproduction is permitted which does not comply with these terms. 